\title{
Voltammetric lability of metal complexes at spherical microelectrodes with various radii.
}

\author{
Josep Galceran ${ }^{\star a}$, Jaume Puy ${ }^{\mathrm{a}}$, José Salvador ${ }^{\mathrm{a}}$, Joan Cecília ${ }^{\mathrm{b}}$ and Herman P. van \\ Leeuwen $^{\mathrm{C}}$
}

a Departament de Química, Universitat de Lleida, Rovira Roure 177, 25198, Lleida, Spain

${ }^{\mathrm{b}}$ Departament de Matemàtica, Universitat de Lleida, Rovira Roure 177, 25198, Lleida, Spain

${ }^{\mathrm{c}}$ Laboratory of Physical Chemistry and Colloid Science, Wageningen University, Dreijenplein 6, 6703 HB Wageningen, The Netherlands

* Corresponding author. e-mail address: galceran@quimica.udl.es

\section{Abstract:}

The size of a microelectrode can have a dramatic impact on the relative importance of the diffusional and kinetic contributions to the voltammetric current of an electroactive metal ion in a complexing medium. Decreasing the radius enhances the diffusional contribution and, as a consequence, the complex system tends to move away from labile behaviour (where an equilibrium relationship holds). Therefore, sufficiently small microelectrodes (either or not combined with short measuring times) should be able to directly sense free metal concentration for not too fast association/dissociation kinetics. The particular case of steady state spherical (or hemispherical) diffusion under ligand excess (pseudo-first order kinetics) is solved analytically. The ensuing lability criterion is shown to be in accordance with a geometrical derivation based on an analysis of the 
random walk of the free metal ions produced by dissociation of the complex. It is shown that, for a generated metal ion, the probability of reaching the microelectrode surface can be quite different from the planar case. Alternatively, the classical reaction layer concept can be used in the derivation of the lability criterion for spherical geometry as it is shown in this work. All treatments quantitatively show how the lability of metal complexes is reduced with decreasing the dimension of the microelectrode.

Keywords: microelectrode, lability, kinetic current, speciation, steady state, reaction layer, CE scheme, pseudo first order, homogeneous complexation.

\section{Introduction}

The dynamic speciation of metals, i.e. the distribution and association/dissociation kinetics of their species, largely governs properties such as reactivities and bioavailabilities in complex systems. It is therefore not surprising that sensitive nonequilibrium techniques, such as voltammetry, are taking a prominent position in this field [1-4]. Voltammetric techniques are generally well-defined in terms of geometrical conditions and operational time-scale. Kinetic qualifications like "labile" and "inert" have an inherently sound theoretical definition, based on comparing limiting fluxes controlled by finite rates of dissociation of complex species to the limiting diffusional fluxes of these species [2]. Results obtained in the voltammetric analysis of the metalligand complexation (such as heavy metals in environmental samples) will be equally indispensable in the interpretation of metal uptake from complex media by microorganisms [5-10]. 
Microelectrodes are defined by dimensions which do not largely exceed the thickness of the operational diffusion layer [11]. Their use is widespread due to several reasons, such as no need of supporting electrolyte, low ohmic drop, attainability of steady state, etc. [12-15]. Due to their geometrical characteristics, the relative importance of the diffusional and kinetic contributions in the mass transport towards a spherical microelectrode can be quite different from the planar case and, thus, the lability of the system will be altered [16,17]. In a first orientational study [18], lability criteria have been worked out by considering two cases i) a spherical diffusion layer and a linear reaction layer and ii) spherical diffusion and reaction layers. This treatment only considered the inequalities involved in lability criteria and was oversimplified in not specifying the metal ion's probabilities of reaching the microelectrode surface from a spherical reaction layer.

Hence, it seems timely to analyse the situation for the microelectrode on a more rigorous level, by studying the properties of the continuity equations describing the evolution of the system (with neither convection nor migration) and extracting the information about the impact of decreasing the electrode dimensions (section 1). In the case of ligand excess (pseudo-first order kinetics) and steady state, the rigorous analytical solution (section 2, with details in the Appendix) for the normalised current is worked out (assuming that no finite volume effects arise from possible saturation inside the sphere [19]). This result leads to a lability criterion (section 2.2.1), which can also be confirmed by an intuitive derivation (section 2.2.2) based on the random walk of metal ions produced by dissociation of complexes close to the electrode surface. Finally (section 2.2.3), the validity of the reaction layer concept in the derivation of the lability criterion for spherical geometry is critically analysed. 


\section{1.- General mathematical formulation .}

Let us consider an electroactive metal ion $\mathrm{M}$

$\mathrm{M} \stackrel{n \mathrm{e}^{-}}{\longrightarrow} \mathrm{M}^{0}$

which is complexed by a ligand $\mathrm{L}$ to yield an electroinactive complex ML, according to the homogeneous reaction:

$M+L=\frac{k_{a}}{\rightleftharpoons} M L$

If we assume that the only relevant mode of transport towards the spherical or hemispherical electrode of radius $r_{0}$ is diffusion, the continuity equations for the species are:

$\frac{\partial c_{M}(r, t)}{\partial t}=D_{M}\left(\frac{\partial^{2} c_{M}(r, t)}{\partial r^{2}}+\frac{2}{r} \frac{\partial c_{M}(r, t)}{\partial r}\right)+k_{d} c_{M L}(r, t)-k_{a} c_{M}(r, t) c_{L}(r, t)$

$\frac{\partial c_{L}(r, t)}{\partial t}=D_{L}\left(\frac{\partial^{2} c_{L}(r, t)}{\partial r^{2}}+\frac{2}{r} \frac{\partial c_{L}(r, t)}{\partial r}\right)+k_{d} c_{M L}(r, t)-k_{a} c_{M}(r, t) c_{L}(r, t)$

and

$\frac{\partial c_{M L}(r, t)}{\partial t}=D_{M L}\left(\frac{\partial^{2} c_{M L}(r, t)}{\partial r^{2}}+\frac{2}{r} \frac{\partial c_{M L}(r, t)}{\partial r}\right)-k_{d} c_{M L}(r, t)+k_{a} c_{M}(r, t) c_{L}(r, t)$

The asssumption $D_{\mathrm{L}}=D_{\mathrm{ML}}$, usual in metal-macromolecular complexation, is not necessary in this work.

Among the boundary conditions we have those for semi-infinite diffusion, prescribing given bulk values at infinite $r$. Thus, far away from the electrode surface or at $t=0$, the bulk concentrations are related by the equilibrium constant: 


$$
K=\frac{k_{a}}{k_{d}}=\frac{C_{M L}^{*}}{C_{M}^{*} C_{L}^{*}}
$$

Boundary conditions at the electrode surface arising from the electroinactivity of complex and ligand are

$$
\left(\frac{\partial \mathrm{c}_{\mathrm{ML}}}{\partial \mathrm{r}}\right)_{\mathrm{r}=\mathrm{r}_{0}}=\left(\frac{\partial \mathrm{c}_{\mathrm{L}}}{\partial \mathrm{r}}\right)_{\mathrm{r}=\mathrm{r}_{0}}=0 \quad \forall \mathrm{t}=0
$$

For the sake of simplicity, we only consider here limiting flux conditions

$$
c_{M}\left(r_{0}, t\right)=0 \quad \forall t \geq 0
$$

and the response function is the limiting current:

$$
I=n F A D_{M}\left(\frac{\partial c_{M}}{\partial r}\right)_{r=r_{0}}
$$

In general, the question of lability concerns the competing relevance of diffusion and complexation kinetics. We now address the question of the impact of the electrode radius $r_{0}$ on the lability.

Let us consider any of the continuity equations (3)-(5), such as the one corresponding to the metal ion, and introduce the change of variable

$$
\rho \equiv \frac{r}{r_{0}}
$$

Omitting the independent variables for simplicity, the equation reads:

$\frac{\partial \mathrm{c}_{\mathrm{M}}}{\partial \mathrm{t}}=\frac{\mathrm{D}_{\mathrm{M}}}{\mathrm{r}_{0}^{2}}\left(\frac{\partial^{2} \mathrm{C}_{\mathrm{M}}}{\partial \rho^{2}}+\frac{2}{\rho} \frac{\partial \mathrm{c}_{\mathrm{M}}}{\partial \rho}\right)+\mathrm{k}_{\mathrm{d}} \mathrm{C}_{\mathrm{ML}}-\mathrm{k}_{\mathrm{a}} \mathrm{C}_{\mathrm{M}} \mathrm{C}_{\mathrm{L}}$

where one can recognise that the diffusion coefficient now combines with $r_{0}^{2}$. This shows that the magnitude of the diffusion terms in the continuity equation increases 
when $r_{0}$ decreases, whereas the kinetic contribution remains without direct $r_{0^{-}}$ dependence (as expected from a volume process). This is consistent with the well known fact that microelectrodes, due to the enhancement of diffusion transport, are particularly useful in tracing fast kinetic processes that become rate limiting under suitable conditions [20]. We conclude that decreasing $r_{0}$ increases the diffusive flux and thus tends to decrease the observed lability of a metal complex in its voltammetric response.

The reasoning can be extended to other microelectrodes, such as the inlaid microdisc, ring or band electrodes. In any case, diffusion is enhanced by the small dimension of the electrode (given by some characteristic length) and lability is lower than in the planar case. As explained at the end of section 2.2.1 these facts might be experimentally exploited to directly determine the bulk concentration of free metal ion.

\section{2.- A particular case: Steady state under excess ligand conditions at a (hemi)spherical electrode.}

\subsection{Analytical solution}

In order to work out a lability criterion for a particularly simple case, we recall that one of the reasons for using microelectrodes lies in their ability for reaching steady state within a reasonable time. Thus, we tackle now the steady state case, which is, of course, simpler than the transient. Consider further that the ligand concentration is so large with respect to the amount of $M$ that its concentration is not altered from the bulk value (ligand excess conditions):

$$
\mathrm{C}_{\mathrm{L}}(\mathrm{r}) \approx \mathrm{C}_{\mathrm{L}}^{*} \quad \forall \mathrm{r}
$$


Then the equilibrium concentrations of metal ion and complex are linearly related by the effective complexation constant $K^{\prime}$ :

$$
C_{M L}(r)=K C_{L}^{*} C_{M}(r)=K{ }^{\prime} C_{M}(r)
$$

We define the non-dimensional (limiting) current as

$$
\phi \equiv \frac{\mathrm{I}\left(\mathrm{C}_{\mathrm{L}}^{*} \neq 0\right)}{\mathrm{I}\left(\mathrm{C}_{\mathrm{L}}^{*}=0\right)}=\frac{\mathrm{r}_{0}}{\mathrm{C}_{\mathrm{T}, \mathrm{M}}^{*}}\left(\frac{\partial \mathrm{c}_{\mathrm{M}}}{\partial \mathrm{r}}\right)_{\mathrm{r}=\mathrm{r}_{0}}
$$

i.e. the ratio between the current corresponding to the system given in eqn. (9) and the current for the same system without ligand,

$$
\mathrm{I}\left(\mathrm{c}_{\mathrm{L}}^{*}=0\right)=\mathrm{nFAD} \mathrm{M}_{\mathrm{M}, \mathrm{M}} \frac{\mathrm{c}_{\mathrm{T}}^{*}}{\mathrm{r}_{0}}
$$

(same amount of total metal $\mathrm{C}_{\mathrm{T}, \mathrm{M}}^{*}$, spherical steady state), being $A$ the electrode area ( $4 \pi r_{0}^{2}$ in the spherical case and half this value for a hemispherical electrode mounted on a planar insulating surface).

As shown in appendix, the normalised current $\phi$ for any kinetic constants can be expressed as:

$$
\phi=\frac{1}{1+\mathrm{K}^{\prime}}\left(1+\frac{\varepsilon \mathrm{K}^{\prime} \sqrt{\kappa_{\mathrm{a}}}}{\sqrt{\varepsilon \mathrm{K}^{\prime}\left(1+\varepsilon \mathrm{K}^{\prime}\right)}+\sqrt{\kappa_{\mathrm{a}}}}\right)
$$

where the ratio of diffusion coefficients $\left(D_{M L} / D_{M}\right)$ is denoted as $\varepsilon$ and $\kappa_{\mathrm{a}}$ is the nondimensional (association) kinetic constant given by:

$$
\kappa_{\mathrm{a}} \equiv \frac{\mathrm{k}_{\mathrm{a}} \mathrm{C}_{\mathrm{L}}^{*} \mathrm{r}_{0}^{2}}{\mathrm{D}_{\mathrm{M}}}
$$

The normalised current for the limiting case of (fully) labile complexation $\left(\kappa_{\mathrm{a}} \rightarrow \infty\right)$ is 


$$
\phi_{\text {labile }}=\frac{1+\varepsilon \mathrm{K}^{\prime}}{1+\mathrm{K}^{\prime}}
$$

while, for inert complexes $\left(\kappa_{\mathrm{a}} \rightarrow 0\right)$ we have

$$
\phi_{\text {inert }}=\frac{1}{1+K^{1}}
$$

Fig 1 shows the behaviour of the non-dimensional current $\phi$ versus the radius (which can be seen as the experimental control for $\kappa_{\mathrm{a}}$ ) for a set of 3 hypothetical systems sharing common values of $\varepsilon, K^{\prime}$ and $D_{\mathrm{M}}$ but with different $k_{\mathrm{d}}$-values. The hypothetical systems could be 3 different metals (if their $D_{\mathrm{M}^{-}}$values were similar enough) with a common ligand, but also the same metal with convenient amounts of 3 different ligands $\left(K^{\prime}=k_{a} C_{L}^{*} / k_{d}\right)$. For all cases, the normalised current increases monotonously with the radius from a minimum value (corresponding to the inert case where $\phi$ is given by (19) ) up to the asymptotic value (corresponding to the labile case of eqn (18) ). As seen in Fig 1, the transition from inert to labile depends both on $r_{0}$ and on the $k_{\mathrm{d}}$ value. Thus, for the system with the highest $k_{\mathrm{d}}$ (more "intrinsically" labile, dotted upper line), the current is practically not affected by kinetics limitations for $r_{0}>100 \mu \mathrm{m}$, while for the system with the lowest $k_{\mathrm{d}}$ (continuous lowest line), the kinetic limitations on the current are still noticeable at $r_{0}=10 \mathrm{~mm}$.

For purposes of comparison, it is convenient to introduce a parameter $\xi$ (ranging from 0 to 1) which could be called "degree of lability " :

$$
\xi \equiv \frac{\phi_{\text {kinetic }}-\phi_{\text {inert }}}{\phi_{\text {labile }}-\phi_{\text {inert }}}=\frac{I_{\text {kinetic }}\left(c_{L}^{*} \neq 0\right)-I_{\text {inert }}\left(c_{L}^{*} \neq 0\right)}{I_{\text {labile }}\left(c_{L}^{*} \neq 0\right)-I_{\text {inert }}\left(c_{L}^{*} \neq 0\right)}
$$

By using the analytical solution (16), one obtains 


$$
\xi=\frac{\sqrt{\kappa_{\mathrm{a}}}}{\sqrt{\varepsilon \mathrm{K}^{\prime}\left(1+\varepsilon \mathrm{K}^{\prime}\right)}+\sqrt{\kappa_{\mathrm{a}}}}
$$

Fig 2 shows $\xi$ for different electrode radii, with parameters typical for $\mathrm{Ni}^{2+}$ complexation [18,21]. It can be seen that at huge electrode radii, say $1 \mathrm{~cm}$ (which would require an impractical time to reach steady state), the lability degree $\xi$ is practically unity, indicating a labile behaviour. On the other hand, for electrode radii less than 10 $\mu \mathrm{m}, \xi$ is less than $5 \%$.

The more labile is a system the larger is the depletion of $c_{\mathrm{ML}}$ close to the electrode surface. In fact, via substitution in the analytical solution for the normalised complex profile (A-13), it can be seen that

$$
\frac{C_{M L}\left(r=r_{0}\right)}{C_{M L}^{*}}=1-\xi
$$

Thus the degree of lability can be seen as an indicator of the lability via the concentration of complex at the electrode surface. If the lability is high, $c_{\mathrm{ML}}\left(r=r_{0}\right)$ is close to zero (see lowest curve in Fig 3). When the system is practically inert, $\mathrm{C}_{\mathrm{ML}}\left(\mathrm{r}=\mathrm{r}_{0}\right) \approx \mathrm{c}_{\mathrm{ML}}^{*}$ (see continuous upper curve in Fig 3$)$.

At this point it is useful to emphasize that if a system is not labile at steady state (due, for instance, to the use of a very small microelectrode), then it is certainly not labile during the transient preceding this steady state.

\subsection{Lability criteria}

\subsubsection{Analytical derivation of a lability criterion}


One could set an arbitrary border-line between predominantly labile systems and predominantly inert systems at $\xi=1 / 2$. This would imply that the first category obeys:

$$
\xi=\frac{\sqrt{\kappa_{\mathrm{a}}}}{\sqrt{\varepsilon \mathrm{K}^{\prime}\left(1+\varepsilon \mathrm{K}^{\prime}\right)}+\sqrt{\kappa_{\mathrm{a}}}}>\frac{1}{2}
$$

which can be written as

$\kappa_{\mathrm{a}}>\varepsilon \mathrm{K}^{\prime}\left(1+\varepsilon \mathrm{K}^{\prime}\right)$

or, in terms of the radius,

$r_{0}>\sqrt{\frac{D_{M L} K^{\prime}\left(1+\varepsilon K^{\prime}\right)}{k_{a} C_{L}^{*}}}$

So, according to this general lability criterion, the radius of the microelectrode has to overcome a minimum value for steady state currents at a (hemi)spherical electrode to be labile. The same conclusion could also be reached from eqn. (23), noticing that, due to $\sqrt{\kappa_{\mathrm{a}}} \propto r_{0}$, the lability degree $\xi$ increases monotonically with increasing $r_{0}$. In any case, it is clear that under the conditions mentioned, the lability of a system only decreases with decreasing radius.

For complexes so strong that $\varepsilon \mathrm{K}^{\prime}>>1$, the general lability criterion (24) simplifies to $\kappa_{\mathrm{a}}>\left(\varepsilon \mathrm{K}^{\prime}\right)^{2}$

(see ref. [22,23] for the similar simplification in the planar case).

After elementary algebraic re-shuffling we obtain, from (26), the following lability criterion:

$$
\frac{k_{d} r_{0}}{D_{M L}} \sqrt{\frac{D_{M}}{k_{a} C_{L}^{*}}}>1
$$


which is just equivalent to expression 13 in reference [18]. Eqn (27) can also be written as a condition for the radius (which could be seen as the experimentally controllable parameter):

$$
r_{0}>\frac{D_{M L}}{\sqrt{D_{M}}} \frac{\sqrt{k_{a} C_{L}^{*}}}{k_{d}}
$$

Let us consider some numerical examples, begining with a system with slow kinetics. With parameters corresponding to $\mathrm{Ni}^{2+}$ complexation taken from figure 4 in ref [18] (used for Fig 2), we can expect (according to eqn. (28)) lability only for radii of the electrode larger than $0.32 \mathrm{~mm}$, which indicates that the system is never labile within the practical microelectrode range. Now, we can compare this with an example of a system with fast kinetics. Parameters suggested for $\mathrm{Pb}^{2+}[18,21]$ are $k_{\mathrm{a}}=10^{10} \mathrm{M}^{-1} \mathrm{~s}^{-1}$ and $D_{\mathrm{M}}=10^{-9}$ $\mathrm{m}^{2} \mathrm{~s}^{-1}$. If the parameters for the ligand are $D_{\mathrm{ML}}=10^{-10} \mathrm{~m}^{2} \mathrm{~s}^{-1}$ and $\mathrm{c}_{\mathrm{L}}^{*}=10^{-5} \mathrm{M}$, application of eqn. (28) predicts that the behaviour of the system can be considered labile for $r_{0}>$ $0.1 \mu \mathrm{m}$.

An experimental exploitation of the results given so far is suggested by eqn. (28): the manipulation of the radius (or the characteristic length of a generic microelectrode) should allow the approximation towards the inert limit. Indeed, a system with not too fast association and dissociation kinetics could be rendered practically inert by using a sufficiently small microelectrode (whichever its geometry and regardless of the fulfilment of the excess ligand conditions, according to the results of section 1). Plots of normalised current against radius would eventually converge towards a flat region (similar to that shown in Fig 1) for sufficiently small microelectrodes. Under these conditions, the current is just due to the free metal concentration and the microelectrode 
could act as a direct free metal ion sensor. Among the application of such detection, one can point out the building up of binding curves for heterogeneous ligands.

\subsubsection{A random walk derivation of a lability criterion}

In order to get a physical picture of the reason why lability always decreases with the electrode radius, we perform here an intuitive derivation of the lability criterion based on the elementary motion of the metal ions close to the microelectrode [24-28]. The key idea is that the number of metal ions that actually reach the surface is substantially lower than in the planar case of a macroscopic electrode.

Let $\mathrm{A}$ be a point in the solution, situated at a distance $R$ of the centre of the electrode (point $\mathrm{O}$ in Fig 4). Let $N$ be the number of particles (free metal ions) present in a infinitesimally small volume around A at a given instant (considered as "initial", when the free metal ions have been just generated and are about to move). We assume:

i) Each of the $N$ particles moves the same distance $\Delta r$ (mean free path) from A, during its life time $\Delta \mathrm{t}_{\mathrm{M}}=\frac{1}{\mathrm{k}_{\mathrm{a}} \mathrm{C}_{\mathrm{L}}^{*}}$. Later on, this distance will be computed from the random walk (or random flight) movement via the Einstein-Smoluchowski relationship

$$
\Delta r=\sqrt{6 D_{M} \Delta t_{M}}
$$

ii) Due to the absence of any privileged direction during the movement, when $\Delta t_{\mathrm{M}}$ has ellapsed, the $N$ particles (originally in A) would (if none of the particles had hit the electrode surface) scatter homogeneously on the surface of the sphere with centre in A and radius $\Delta r$ (this "diffusion sphere" is shown in Fig 4 in light grey). 
iii) The mean number of particles that hit the electrode surface is counted as if the "trajectories" of the particles leaving A were straight lines (radii from the "diffusion sphere" with centre in A). Those radii intercepting the electrode surface are considered to represent the trajectories of particles hitting the electrode surface.

From the previous hypotheses, it follows that the fraction of particles hitting the electrode surface is proportional to the area of the projection or "shadow" of the electrode surface on the "diffusion sphere". This area (in Fig 4, see dotted arc on the "diffusion sphere" drawn in light grey) is generated by those particles that move away from A following a radius and are intercepted by the electrode surface (dark grey upper circle). Notice that in Fig 4 the 2-D dotted arc is the section of the "shadowed" casket (3-D, not drawn), whose area is the relevant one. Then, the fraction of particles hitting the electrode surface (or probability of hit $p_{\text {hit }}$ ), can be computed as a ratio of areas:

$$
p_{\text {hit }}=\frac{\text { "shadowed "casket area }}{\text { diffusion spherearea }}
$$

Depending on the relationship between $R, \Delta r$ and $r_{0}$, two cases arise. The first case, which could be called "small shielding case" arises when no radius of the "diffusion sphere" pointing to the shadowed casket area is tangent to the electrode surface and is depicted in Fig 4. For this case, elementary geometrical reasonings lead to:

$$
\mathrm{p}_{\text {hit,small shielding }}=\frac{1}{2}-\frac{\mathrm{R}}{4 \Delta \mathrm{r}}+\frac{\left(\mathrm{r}_{0}^{2}-\Delta \mathrm{r}^{2}\right)}{4 \mathrm{R} \Delta \mathrm{r}} \quad \text { for } \quad \Delta \mathrm{r}^{2}+\mathrm{r}_{0}^{2}<\mathrm{R}^{2}
$$

The second case, depicted in Fig 5, could be called "large shielding". The projection of the electrode surface on the "diffusion sphere" includes all the radius in between the extreme cases of tangent radii. For this case: 


$$
\mathrm{p}_{\text {hit,largeshielding }}=\frac{1}{2}\left[1-\sqrt{1-\frac{\mathrm{r}_{0}^{2}}{\mathrm{R}^{2}}}\right] \quad \text { for } \quad \Delta \mathrm{r}^{2}+\mathrm{r}_{0}^{2} \geq \mathrm{R}^{2}
$$

where no dependence on $\Delta r$ appears, as $p_{\text {hit }}$ is just related to the solid angle with which the whole electrode is seen from A. In particular, if A lies just on the electrode surface, $1 / 2$ of the particles would hit the electrode, as expected for planar diffusion. But, when A moves away from the electrode surface the probability of the particles hitting the electrode decreases as some of them follow radii not intercepting its surface. These results answer the key question: due to the spherical diffusion not all the particles (nor just $1 / 2$ of them) at a given $R<\Delta r+r_{0}$ hit the electrode surface, but there is a functional dependence involving $R, \Delta r$ and $r_{0}$.

Let us now derive the kinetic flux $J_{\text {kin }}$ from the hitting probabilities just found. For simplicity, we assume a uniform $\mathrm{C}_{\mathrm{ML}}^{*}$ for all spatial points (this will overestimate $J_{\text {kin }}$ in a labile case as seen in see Fig 3). The number of moles $n_{\text {hit }}$ of kinetically generated M crossing the electrode surface per unit of time can, then, be computed as:

$$
n_{\text {hit }}=k_{d} C_{M L}^{*}\left[\int_{r_{0}}^{\sqrt{\Delta r^{2}+r_{0}^{2}}} p_{\text {hit,largeshielding }} 4 \pi R^{2} d R+\int_{\sqrt{\Delta r^{2}+r_{0}^{2}}}^{r_{0}+\Delta r} p_{\text {hit,small shielding }} 4 \pi R^{2} d R\right]
$$

Using expressions (31) and (32) for the probability and integrating, we find

$$
\mathrm{n}_{\text {hit }}=\mathrm{k}_{\mathrm{d}} \mathrm{C}_{\mathrm{ML}}^{*} \pi \mathrm{r}_{0}^{2} \Delta \mathrm{r}
$$

Thus, the kinetic flux can be computed as:

$$
\mathrm{J}_{\text {kin }}=\frac{\mathrm{n}_{\text {hit }}}{4 \pi \mathrm{r}_{0}^{2}}=\frac{1}{4} \Delta r \mathrm{k}_{\mathrm{d}} \mathrm{C}_{\mathrm{ML}}^{*}
$$

which indicates that the kinetic flux is independent of $r_{0}$. Notice that the expression $n_{\text {hit }}=k_{d} c_{M L}^{*} \frac{4 \pi}{3}\left[\left(r_{0}+\Delta r\right)^{3}-r_{0}^{3}\right]$ cannot be used, except for the case $\Delta r<<r_{0}$. 
On the other hand, the diffusional flux

$$
J_{\text {dif }}=\frac{D_{M L} C_{M L}^{*}}{r_{0}}
$$

clearly increases monotonically with decreasing $r_{0}$.

The lability condition could be formulated by imposing [2,23,29] a higher kinetic flux $\left(J_{\text {kin }}\right)$ than diffusional one $\left(J_{\text {dif }}\right)$. Using eqn (29) for $\Delta r$ with $\Delta \mathrm{t}_{\mathrm{M}}=\frac{1}{\mathrm{k}_{\mathrm{a}} \mathrm{C}_{\mathrm{L}}^{*}}$ in the expression of $J_{\text {kin }}(35)$, the result is

$$
r_{0}>\frac{4}{\sqrt{6}} \frac{D_{M L}}{\sqrt{D_{M}}} \frac{\sqrt{k_{a} C_{L}^{*}}}{k_{d}}
$$

which only differs from the analytically found expression (28) by a numerical coefficient close to unity. The criterion again indicates that decreasing $r_{0}$ always diminishes lability.

\subsubsection{Derivation of the lability criterion using the reaction layer concept}

As in some derivations of lability criteria the reaction layer concept has been used, let us revisit now this issue. Several definitions of reaction layer [30-33] have been given. In this work, we adhere to the operational concept of reaction layer, based on the decomposition of the total current into a "free metal contribution (inert)" and a "kinetic current", which can be expressed in terms of fluxes as:

$J_{\text {total }}=J_{\text {inert }}+J_{\text {kin }}$

The thickness of the reaction layer $\mu$ is a kinetic parameter [34-39], defined such that:

$$
\mathrm{J}_{\mathrm{kin}}=\mathrm{C}_{\mathrm{ML}}^{*} \mathrm{k}_{\mathrm{d}} \mu
$$


In steady state spherical diffusion, the results from random walk analysis in section 2.2.2 support the interpretation of $\mu$ as the mean distance travelled by the free metal ion during its life time $\Delta t_{\mathrm{M}}$ [27,32]: it suffices to compare eqns (35) and (39) while neglecting numeric factors (close to 1 ) . Thus, the reaction layer can be physically understood as the region from where the electroactive species generated due to the complex dissociation is able to contribute to the current [34].

The computation of $\mu$ for steady-state spherical diffusion can be done, for the simplest case, following the procedure used in planar diffusion [33] with the appropriate corrections. The key difference is that the spherical geometry can maintain a steady state for $c_{M}$ based on diffusion alone $\left(J\right.$ inert $\left.=D_{M} C_{M}^{*} / r_{0}\right)$, while the planar cannot (i.e. in planar geometry all the current is a kinetic current as $J_{\text {iner }}=0$ in steady state for semiinfinite diffusion as the sole transport mode). For simplicity, we use excess of ligand and a uniform $\mathrm{C}_{\mathrm{ML}}^{*}$ for all spatial points (as done for the random walk derivation of the lability criterion). Solving equation (3) for $c_{\mathrm{M}}$ with $\mathrm{C}_{\mathrm{L}}(r) \approx \mathrm{C}_{\mathrm{L}}^{*}$ and $\mathrm{C}_{\mathrm{ML}}(r) \approx \mathrm{C}_{\mathrm{ML}}^{*}$ under steady state conditions $\left(\partial \mathrm{c}_{\mathrm{M}} / \partial \mathrm{t}=0\right)$, we obtain

$C_{M}=c_{M}^{*}\left(1-\frac{r_{0}}{r} e^{-\sqrt{\frac{k_{C} C_{L}^{*}}{D_{M}}}\left(r-r_{0}\right)}\right)$

from which the flux can be computed as:

$$
J_{\text {total }}=D_{M}\left(\frac{\partial c_{M}}{\partial r}\right)_{r=r_{0}}=D_{M} C_{M}^{*}\left(\frac{1}{r_{0}}+\sqrt{\frac{k_{a} C_{L}^{*}}{D_{M}}}\right)
$$


Now we can identify the terms corresponding to the contribution due to pure spherical diffusion of $c_{\mathrm{M}}$ and the kinetic contribution due to the dissociation of $c_{\mathrm{ML}}$ along the reaction layer $\left(J_{k i n}\right)$ :

$J_{\text {total }}=\frac{D_{M} C_{M}^{*}}{r_{0}}+D_{M} C_{M}^{*} \sqrt{\frac{k_{a} C_{L}^{*}}{D_{M}}}=J_{\text {inert }}+c_{M L}^{*} k_{d} \sqrt{\frac{D_{M}}{k_{a} C_{L}^{*}}}=J_{\text {inert }}+J_{\text {kin }}$

By comparison with the operational definition (39), we find that the thickness of the diffusion layer for steady state spherical diffusion coupled with a first order homogeneous reaction is

$\mu=\sqrt{\frac{\mathrm{D}_{\mathrm{M}}}{\mathrm{k}_{\mathrm{a}} \mathrm{C}_{\mathrm{L}}^{*}}}$

in coincidence with the same well-known result $[32,33]$ for planar symmetry, excess ligand and $\varepsilon K^{\prime}>1$. This value for $\mu$, combined with the diffusional flux expression (36), has been successfully used [18] in the derivation of the lability criterion (28) (found from the analytical solution for the current). However, it must be pointed out that in steady state spherical diffusion, regardless of the relative sizes of $\mu$ and $r_{0}$, the kinetic flux must be computed combining eqns (39) and (43).

\section{Conclusions}

From inspection of the continuity equations of the metal-ligand system in spherical geometry, it has been concluded that diffusion towards the microelectrode is enhanced when the electrode radius $r_{0}$ decreases, while the kinetic terms are unaltered. Thus, a decrease in $r_{0}$ favours the kinetic contribution and tends to reduce the lability of the metal complex system. This result can also be applied to microelectrodes other than the spherical or hemispherical ones, on which the discussion has been focused. For not too fast kinetics of complexation, the free metal ion concentration might be directly sensed 
using sufficiently small microelectrodes from normalised currents at a flat region in the plot $\phi$ vs $r_{0}$.

The analytical solution of the problem for steady state spherical diffusion can be derived for the ligand excess case (see Appendix). An important result is that the normalised current depends only on three parameters: $\varepsilon, K^{\prime}$ and $\kappa_{\mathrm{a}}$ (see eqn. (A-7)). The latter parameter (given by eqn (17)) is the non-dimensional rate constant and includes $r_{0}^{2}$ as a factor multiplying $k_{\mathrm{a}}$, thus expressing that in terms of kinetic effects a decrease in $r_{0}$ is equivalent to a decrease in $k_{\mathrm{a}}$. From the analytical expression for the normalised current (see eqn. (16)), a rigorous lability criterion (eqn (24) ) is found, which (for large $\varepsilon K^{\prime}$ ) is equivalent to the one already previously found in the literature. Practically the same criterion (see eqn. (37)) can also be obtained from an alternative derivation making use of a statistical random walk approach. Such analysis demonstrates that the probability of reaching the electrode surface depends on the position of the metal ion (produced from dissociation of complex) with respect to the electrode surface. For spherical geometry, steady state, excess of ligand, pseudo first order reaction and no complex depletion, the expression for the reaction layer thickness $\mu$ (43) is the same as the well-known expression for planar geometry. The reaction layer concept can be used to successfully derive a lability criterion which is essentially the same as that obtained with the analytical solution (27) or the random walk approach (37).

\section{Acknowledgements}

The authors gratefully acknowledge support of this research by the Spanish Ministry of Education and Science (DGICYT: Project BQU2000-0642), from the "Ajuntament de Lleida" and from the "Comissionat d'Universitats i Recerca de la Generalitat de Catalunya". An Iberdrola professorship to HvL is also acknowledged. 


\section{Appendix: Steady state current for the "kinetic" case under spherical diffusion and ligand excess.}

We need to solve the steady state analogues of eqns. (3)-(5) with no-time dependency and the ligand excess condition (12). In fact, the latter condition renders the continuity equation (4) unnecessary and implies the crucial linerarisation of the remaining equations (for the bulk concentrations see eqn. (13)).

Apart from (10), we introduce the following dimensionless variables:

$$
\theta \equiv \frac{\mathrm{C}_{\mathrm{M}}}{\mathrm{C}_{\mathrm{M}}^{*}} \quad \lambda \equiv \frac{\mathrm{C}_{\mathrm{ML}}}{\mathrm{C}_{\mathrm{ML}}^{*}}
$$

where the spatial dependence (such as $\theta(\rho))$ is not explicited.

With these dimensionless variables, eqn. (3) for steady-state can be written:

$$
0=\frac{1}{\rho^{2}} \frac{d}{d \rho}\left(\rho^{2} \frac{d \theta}{d \rho}\right)+\frac{k_{a} C_{L}^{*} r_{0}^{2}}{D_{M}}(\lambda-\theta)
$$

which suggests the introduction of the dimensionless kinetic constant $\kappa_{\mathrm{a}}$ given by (17).

Summation of eqns.(A-2) and (5) (using the dimensionless variables) cancels out the kinetic terms:

$$
0=\frac{1}{\rho^{2}} \frac{\mathrm{d}}{\mathrm{d} \rho}\left(\rho^{2} \frac{\mathrm{d}}{\mathrm{d} \rho}\left(\theta+\varepsilon \mathbf{K}^{\prime} \lambda\right)\right)
$$

The boundary conditions are:

$$
\begin{array}{lll}
\rho=1 & \theta=0 & \frac{\mathrm{d} \lambda}{\mathrm{dx}}=0 \\
\rho \rightarrow \infty & \theta=1 & \lambda=1
\end{array}
$$

The magnitude sought is $\phi$, given by (14), which can be expressed:

$$
\phi=\frac{1}{1+K^{\prime}}\left(\frac{d \theta}{d \rho}\right)_{\rho=1}
$$

From this re-formulation of the problem, it is easy to deduce that the solution depends just on 3 parameters: 
$\phi=\phi\left(\varepsilon, \mathrm{K}^{\prime}, \kappa_{\mathrm{a}}\right)$

The solution of eqn. (A-3), taking into account the boundary values, is:

$$
\theta+\varepsilon \mathrm{K}^{\prime} \lambda=-\frac{1}{\rho}\left(\frac{\mathrm{d} \theta}{\mathrm{d} \rho}\right)_{\rho=1}+1+\varepsilon \mathrm{K}^{\prime}
$$

Substitution of the expression of $\lambda$ (isolated from (A-8)) into (A-2), and introduction of the new variable $\omega=\rho \theta$, yields

$$
\frac{\mathrm{d}^{2} \omega}{\mathrm{d} \rho^{2}}-\kappa_{\mathrm{a}}\left(\frac{1}{\varepsilon \mathrm{K}^{\prime}}+1\right) \omega-\frac{\kappa_{\mathrm{a}}}{\varepsilon \mathrm{K}^{\prime}}\left(\frac{\mathrm{d} \theta}{\mathrm{d} \rho}\right)_{\rho=1}+\kappa_{\mathrm{a}}\left(\frac{1}{\varepsilon \mathrm{K}^{\prime}}+1\right) \rho=0
$$

Its solution, in terms of 2 integrations constants $C_{1}$ and $C_{2}$ is:

$$
\omega=\mathrm{C}_{1} \mathrm{e}^{-\sqrt{\kappa_{\mathrm{a}}\left(\frac{1}{\varepsilon \mathrm{K}^{\prime}}+1\right)} \rho}+\mathrm{C}_{2} \mathrm{e}^{\sqrt{\kappa_{\mathrm{a}}\left(\frac{1}{\varepsilon \mathrm{K}^{\prime}}+1\right)} \rho}-\frac{1}{1+\varepsilon \mathrm{K}^{\prime}}\left(\frac{\mathrm{d} \theta}{\mathrm{d} \rho}\right)_{\rho=0}+\rho
$$

As $\theta$ is bounded, $C_{2}=0$. The boundary condition $\theta=0$ at $\rho=1$ requires

$$
C_{1}=\frac{\left(\frac{\mathrm{d} \theta}{\mathrm{d} \rho}\right)_{\rho=1}-\left(1+\varepsilon \mathrm{K}^{\prime}\right)}{\left(1+\varepsilon \mathrm{K}^{\prime}\right) \mathrm{e}^{-\sqrt{\kappa_{\mathrm{a}}\left(\frac{1}{\varepsilon \mathrm{K}^{\prime}+1}\right)}}}
$$

Computing $\left(\frac{\mathrm{d} \theta}{\mathrm{d} \rho}\right)_{\rho=1}$ from the newly found solution, one can finally obtain:

$$
\left(\frac{\mathrm{d} \theta}{\mathrm{d} \rho}\right)_{\rho=1}=1+\frac{\varepsilon \mathrm{K}^{\prime} \sqrt{\kappa_{\mathrm{a}}}}{\sqrt{\varepsilon \mathrm{K}^{\prime}\left(1+\varepsilon \mathrm{K}^{\prime}\right)}+\sqrt{\kappa_{\mathrm{a}}}}
$$

which, through substitution into (A-6), leads to (16).

The same result can be derived from the work of Oldham [40], imposing zero flux for one of the "interconverting isomers" (see eqn. (18) in ref. [40]) and zero concentration for the other interconverting isomer. 
After some algebra, it can also be seen that the normalised profile of complex is:

$\lambda=1-\frac{\sqrt{\kappa_{\mathrm{a}}}}{\rho\left(\sqrt{\varepsilon \mathrm{K}^{\prime}\left(1+\varepsilon \mathrm{K}^{\prime}\right)}+\sqrt{\kappa_{\mathrm{a}}}\right)}-\sqrt{\frac{\varepsilon \mathrm{K}^{\prime}}{1+\varepsilon \mathrm{K}^{\prime}}} \frac{\left(1-\mathrm{e}^{-(\rho-1) \sqrt{\frac{\kappa_{\mathrm{a}}\left(1+\varepsilon \mathrm{K}^{\prime}\right)}{\varepsilon \mathrm{K}^{\prime}}}}\right)}{\rho\left(\sqrt{\varepsilon \mathrm{K}^{\prime}\left(1+\varepsilon \mathrm{K}^{\prime}\right)}+\sqrt{\kappa_{\mathrm{a}}}\right)}(\mathrm{A}-13)$

\section{References}

[1] J. Buffle, Complexation Reactions in Aquatic Systems. An Analytical Approach., Ellis Horwood Limited, Chichester, 1988, Chapter 9, p. 467.

[2] H.P. van Leeuwen, J. Buffle and R. Cleven, Pure Appl. Chem. 61 (1989) 255.

[3] A.M. Mota and M.M. Correia dos Santos, in A.Tessier and D. R. Turner (Eds.), Metal Speciation and Bioavailability in Aquatic Systems, vol 3, John Wiley \& Sons, Chichester, 1995,

[4] J. Buffle and M.L. TercierWaeber, in J. Buffle and G. Horvai (Eds.), In Situ Monitoring of Aquatic Systems. Chemical Analysis and Speciation, vol 6, John Wiley \& Sons, Chichester, 2000, p. 279.

[5] G.A. Jackson and J.J. Morgan, Limnol. Oceanogr. 23 (1978) 268.

[6] M. Whitfield and D.R. Turner, in B. A. Jenne (Ed.), Chemical Modeling in Aqueous Systems, ACS, Washington DC, 1979, p. 657.

[7] A. Tessier, J. Buffle and P.G.C. Campbell, Chemical and Biological Regulation of Aquatic Systems, Lewis Publishers, Boca Raton, 1994, Chapter 6, p. 197.

[8] R.J.M. Hudson, Sci. Total Envir. 219 (1998) 95.

[9] H.P. van Leeuwen, Environ. Sci. Technol. 33 (1999) 3743.

[10] J.P. Pinheiro and H.P. van Leeuwen, Environ. Sci. Technol. 35 (2001) in press.

[11] K. Stulik, C. Amatore, K. Holub, V. Marecek and W. Kutner, Pure Appl. Chem. 72 (2000) 1483.

[12] R.M. Wightman and D.O. Wipf., in A.J. Bard (Ed.) Electronalytical Chemistry vol 15, Marcel Dekker, New York, 1989, p.267.

[13] M.I. Montenegro, M.A. Queiros and J.L. Daschbach (Eds.). Microelectrodes: Theory and Applications. NATO ASI Series E. Vol. 197, Kluwer, Dordrecht, 1991. 
[14] D. Pletcher, in M. I. Montenegro, M.A. Queiros and J.L. Daschbach (Eds.), Microelectrodes: Theory and Applications. NATO ASI Series E. Vol. 197, Kluwer, Dordrecht, Kluwer, 1991, p. 3.

[15] K.B. Oldham, in M.I. Montenegro, M.A. Queiros and J.L. Daschbach (Eds.), Microelectrodes: Theory and Applications. NATO ASI Series E. Vol. 197, Kluwer, Dordrecht, Kluwer, 1991, p. 35.

[16] T. Carofiglio, F. Magno and I. Lavagnini, J. Electroanal. Chem. 373 (1994) 11.

[17] S. Daniele, C. Bragato and M.A. Baldo, J. Electroanal. Chem. 439 (1997) 153.

[18] H.P. van Leeuwen and J.P. Pinheiro, J. Electroanal. Chem. 471 (1999) 55.

[19] J. Galceran, J. Salvador, J. Puy, F. Mas, D. Gimenez and M. Esteban, J. Electroanal. Chem. 442 (1998) 151.

[20] K.B. Oldham, J.C. Myland, Fundamentals of Electrochemical Science, Academic Press, San Diego, 1994.

[21] F.M.M. Morel and J.G. Hering, Principles and Applications of Aquatic Chemistry, John Wiley, New York, 1993, Chapter 6,

[22] H.G. de Jong, K.Holub and H.P. van Leeuwen, J. Electroanal. Chem. 234 (1987) 1.

[23] H.G. de Jong and H.P. van Leeuwen, J. Electroanal. Chem. 234 (1987) 17.

[24] S. Chandrasekhar, Rev. Modern Phys. 15 (1943) 1.

[25] P. Delahay, J. Amer. Chem. Soc. 73 (1951) 4944.

[26] E.F. Orlemann and D.M.H. Kern, J. Amer. Chem. Soc. 75 (1953) 3058.

[27] E. Budewski, Compt. rend. Acad. Bulg. Sci. 8 (1955) 25.

[28] O.S. Andersen and S.W. Feldberg, J. Phys. Chem. 100 (1996) 4622.

[29] W. Davison, J. Electroanal. Chem. 87 (1978) 395.

[30] P. Delahay, New Instrumental Methods in Electrochemistry, Interscience, New York, 1954.

[31] J. Koutecky and J.Koryta, Electrochim. Acta 3 (1961) 318.

[32] J. Heyrovsky, J.Kuta, Principles of Polarography, Academic Press, New York, 1966.

[33] J. Koryta, J. Dvorak and L. Kavan, Principles of Electrochemistry, Second ed. John Wiley, Chichester, 1993.

[34] J. Koutecky and R. Brdicka, Collect. Czech. Chem. Commun. 12 (1947) 337. 
[35] A.J. Bard, L.R. Faulkner, Electrochemical Methods, Fundamentals and Applications, Wiley, New York, 1980.

[36] G.L. Che and S.J. Dong, Electrochim. Acta. 37 (1992) 2695.

[37] G.L. Che and S.J. Dong, Electrochim. Acta. 37 (1992) 2701.

[38] Q.K. Zhuang and H.Y. Chen, J. Electroanal. Chem. 346 (1993) 29.

[39] Q.K. Zhuang and D.D. Sun, J. Electroanal. Chem. 440 (1997) 103.

[40] K.B. Oldham, J. Electroanal. Chem. 313 (1991) 3. 


\section{Figure Captions}

Fig 1 : Impact of the rate constants on the lability. Plot of the normalised current $(\phi$ given by (16)) expected for three systems sharing $\varepsilon=0.1, K^{\prime}=10^{2}$ and $D_{\mathrm{M}}=10^{-9} \mathrm{~m}^{2} \mathrm{~s}^{-1}$ vs. the logarithm of the electrode radius $r_{0}$. Solid line: $k_{\mathrm{d}}=10^{-2} \mathrm{~s}^{-1}$; dashed line: $k_{\mathrm{d}}=1 \mathrm{~s}^{-1}$; dotted line: $k_{\mathrm{d}}=10^{2} \mathrm{~s}^{-1}$.

Fig 2 : Plot of the "degree of lability " $\xi$ (see eqn (20)) vs. the electrode radius $r_{0}$ (notice its logarithmic scale). Data corresponding to $\mathrm{Ni}^{2+}$ complexation (see figure 4 in $\operatorname{ref}[18]): \varepsilon K^{\prime}=10, D_{\mathrm{M}}=10^{-9} \mathrm{~m}^{2} \mathrm{~s}^{-1} ; k_{\mathrm{a}}=10^{5} \mathrm{M}^{-1} \mathrm{~s}^{-1}$ and $\mathrm{C}_{\mathrm{L}}^{*}=10^{-5} \mathrm{M}$

Fig 3 : Impact of the lability on the normalised profiles of complex $\left(\lambda \equiv \mathrm{C}_{\mathrm{ML}} / \mathrm{C}_{\mathrm{ML}}^{*}\right.$ versus $\rho \equiv \mathrm{r} / \mathrm{r}_{0}$ ) with data as in Fig 2 . Continuous upper line: $r_{0}=10 \mu \mathrm{m}$ (yielding $\xi \approx 0.03$ ); dashed line: $r_{0}=0.1 \mathrm{~mm}$ (yielding $\xi \approx 0.23$ ) and dotted lower curve: $r_{0}=1 \mathrm{~mm}$ (yielding $\xi \approx 0.75$ ). Notice the relationship (26) between $\xi$ and the concentration of complex at the electrode surface. $\lambda$ has been computed analytically using the solution $(\mathrm{A}-13)$. 
Fig 4 : Schematic representation (in a planar section) of the diffusion of $N$ particles from A towards the electrode surface (upper small circle of radius $r_{0}$, drawn with continuous line). The fraction of particles hitting the electrode surface is assumed to be proportional to the area (shown with "bullets") projected by the electrode on the "diffusion sphere" (large circle of radius $\Delta r$ drawn with dashed line ).

Fig 5 : Case of "large shielding": the electrode surface intercepts a relatively large amount of radii. Same conventions as in Fig 4. A radius of the "diffusion sphere" tangent to the electrode surface is also depicted. 


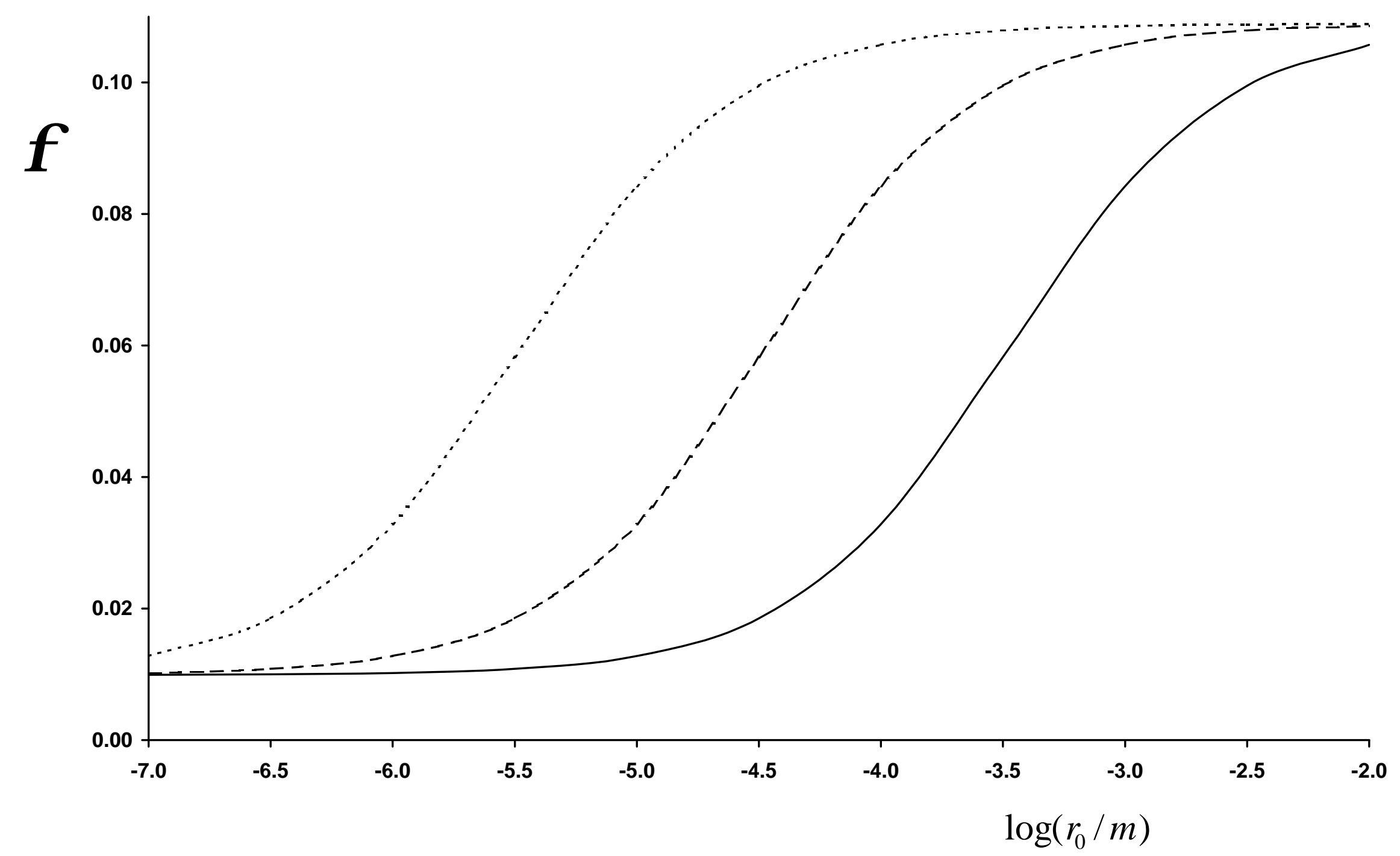

Fig 1 


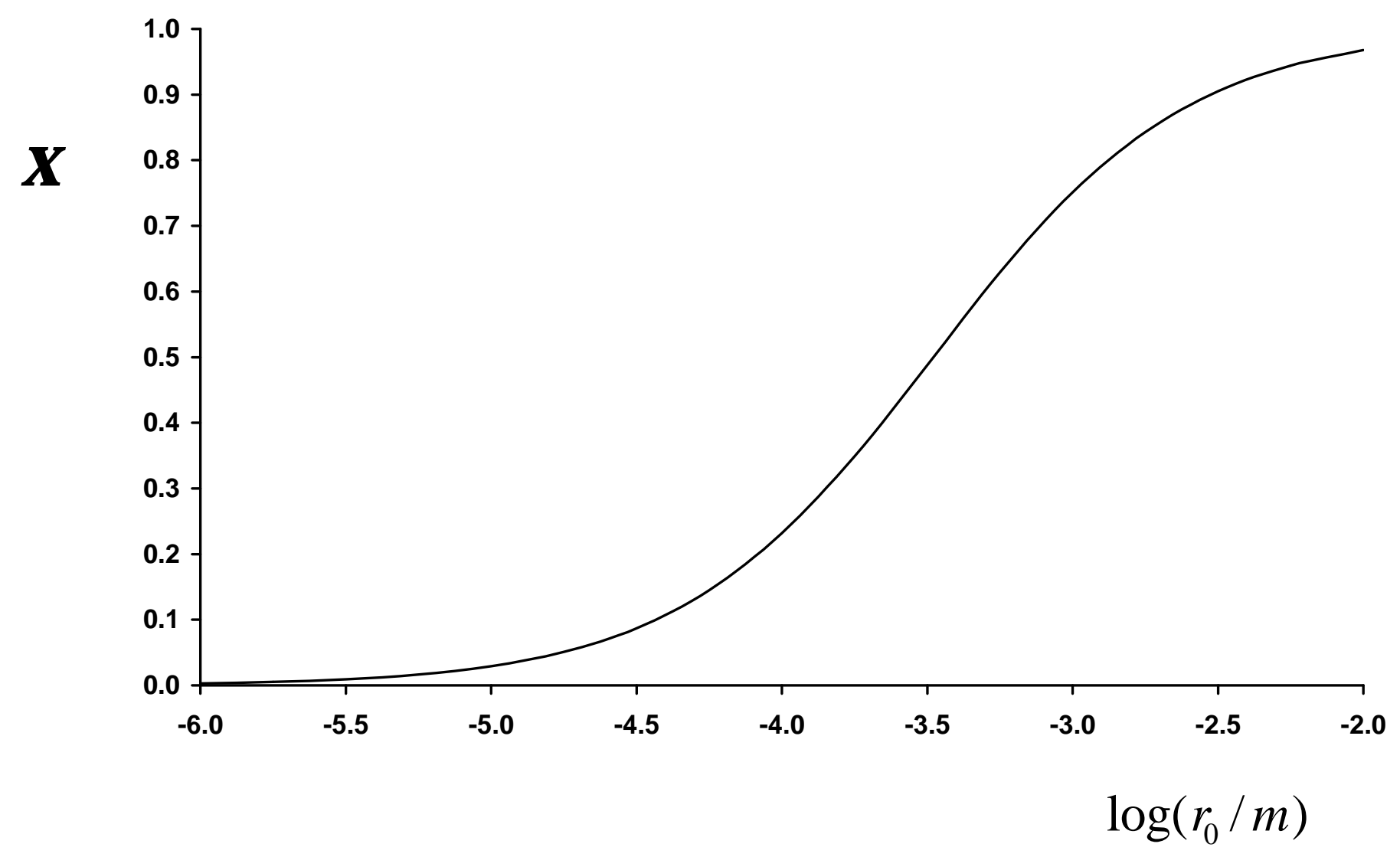

Fig 2 


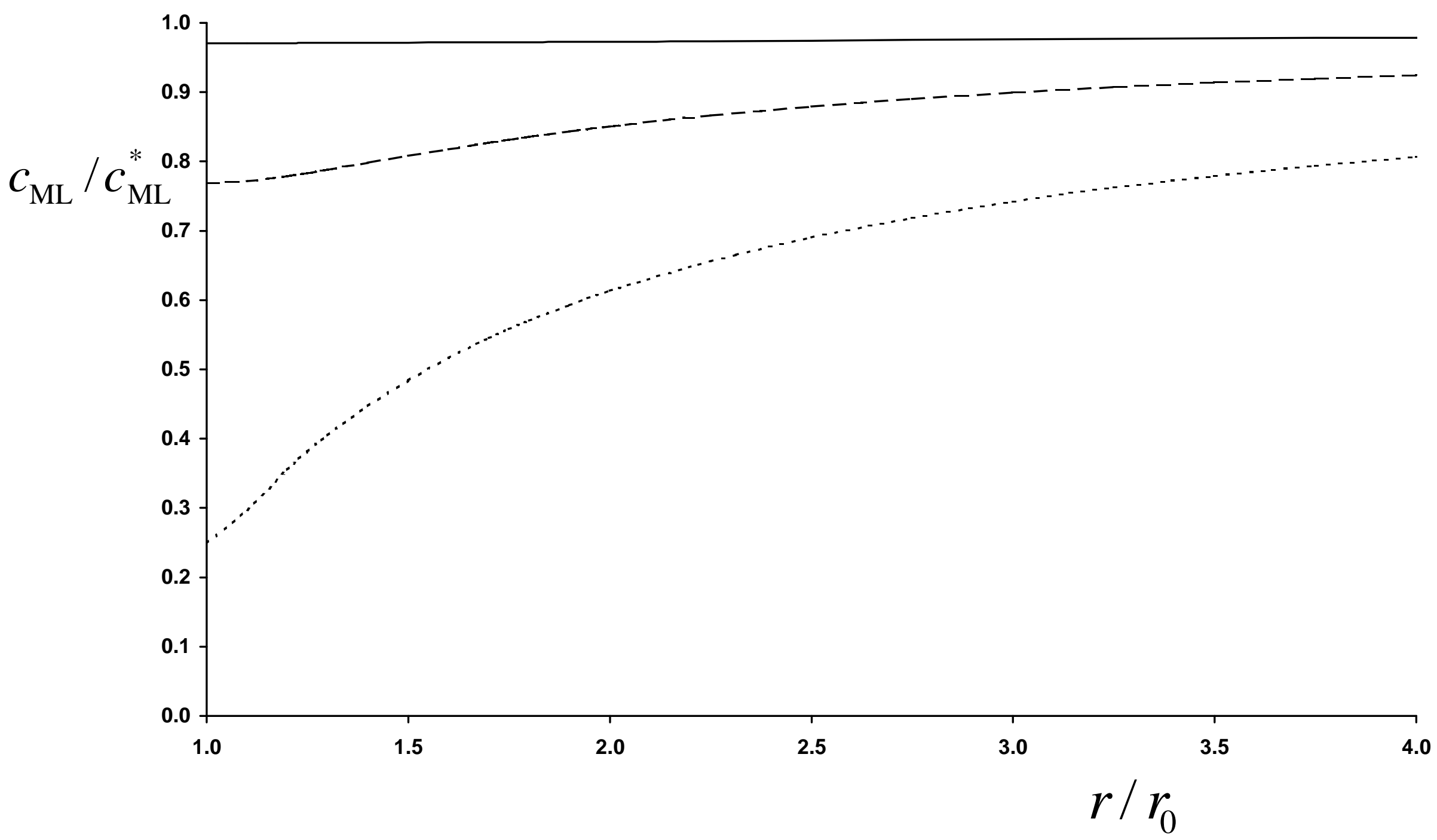

Fig 3 


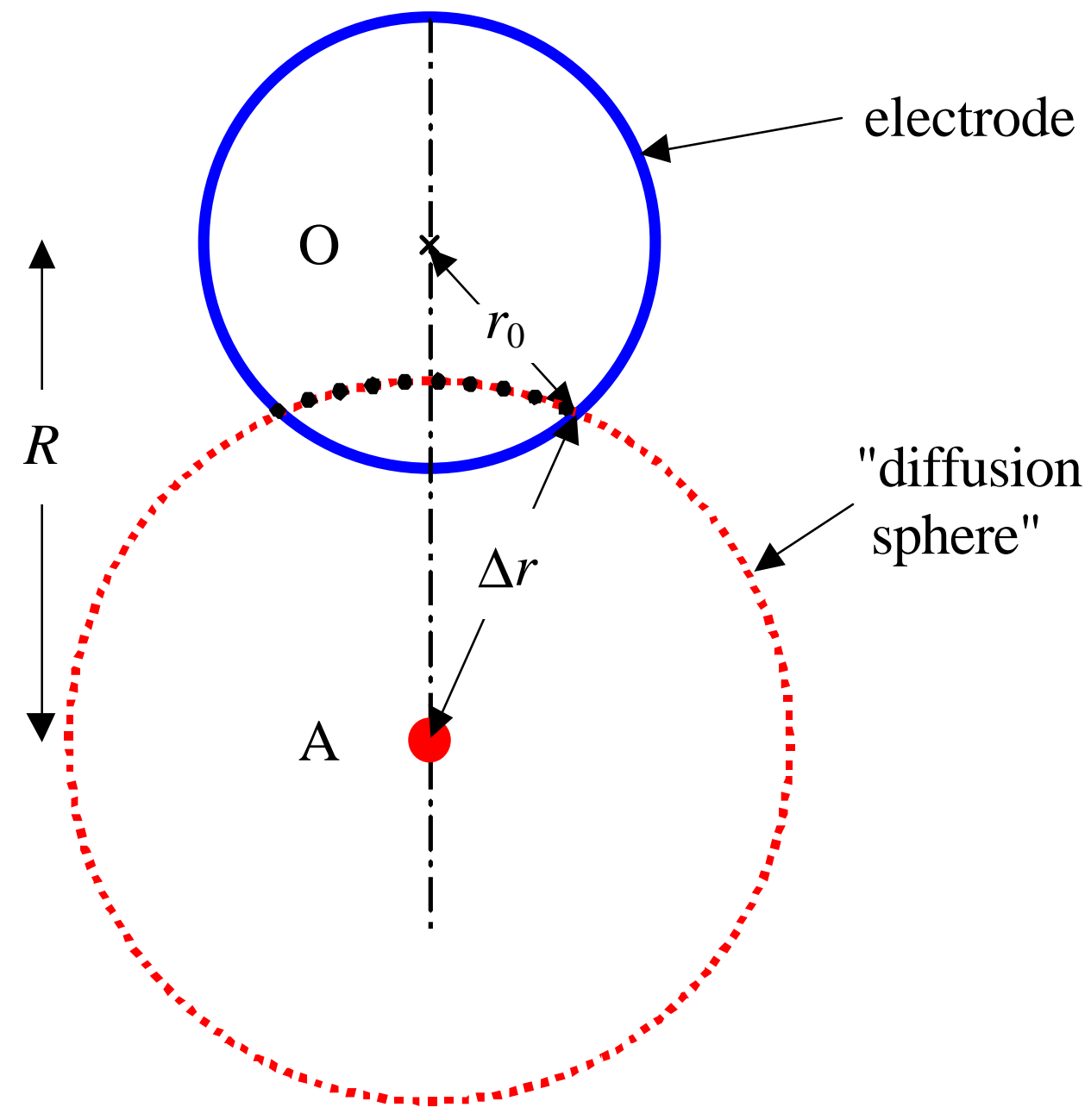

Fig 4 


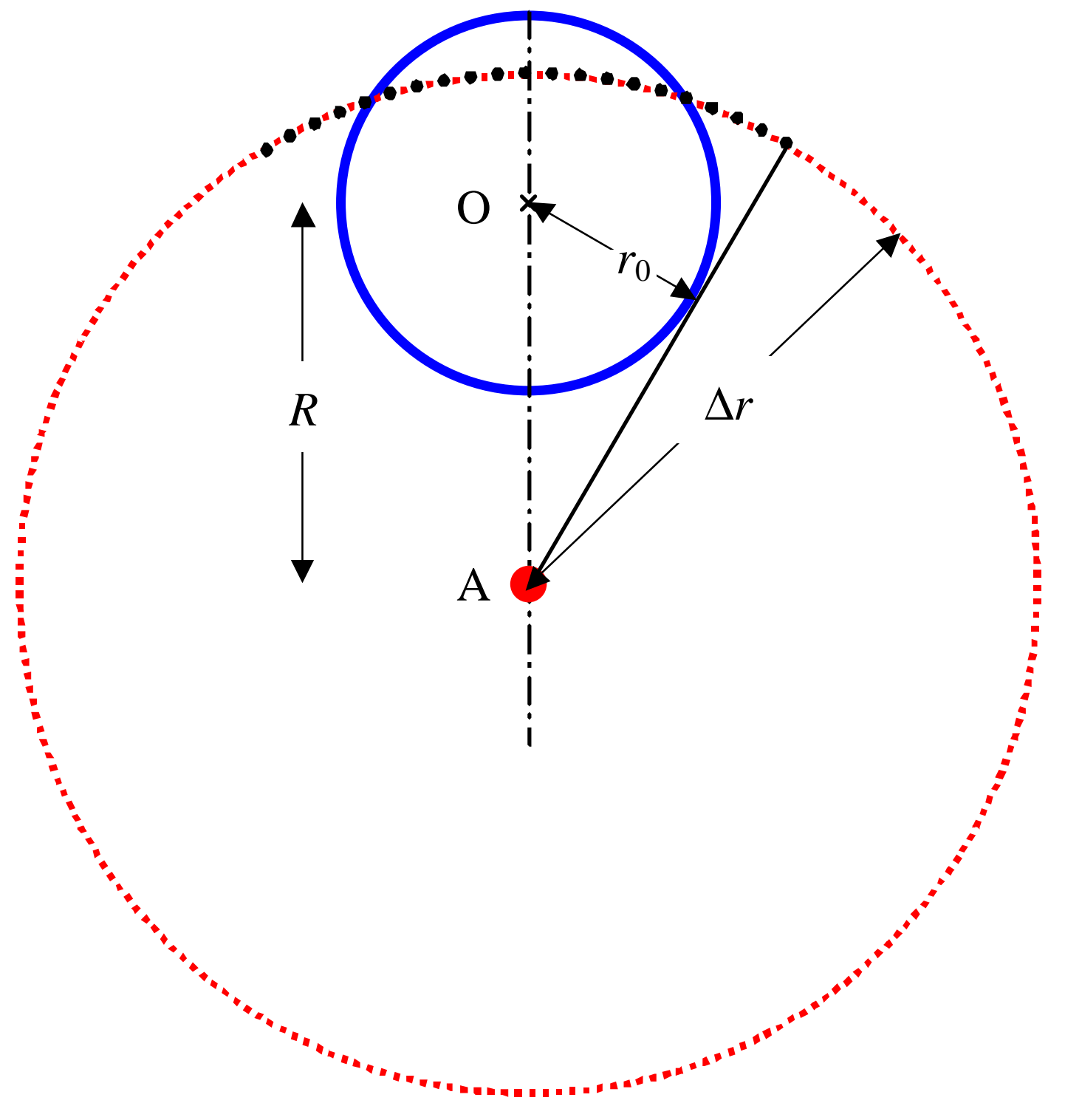

Fig 5 